\title{
Expert System Source Identification of Excessive Vibration
}

\author{
R. Gordon Kirk and Zenglin Guo \\ Virginia Polytechnic Institute and State University, Blacksburg, Virginia, USA
}

The importance of vibration data in determining the condition of rotating machinery is well established in both the aircraft and the heavy equipment industries. Installation of noncontact displacement probes for shaft motion and either velocity or acceleration sensors for bearing cap or foundation motion is standard practice for equipment manufacturers. Automation of the diagnostic evaluation of certain simple faults can be easily implemented. The advances in computer languages in recent years have made it difficult to keep pace with the graphical capabilities available to the programmers. One major concern is the content of the knowledge base and the method of modifying the knowledge base or the procedure of evaluating the confidence of a given identified possible cause of a problem. This article addresses the application of one such expert system to a recent vibration problem on a 7-megawatt steam turbine-driven generator located at the power plant of Virginia Polytechnic Institute.

Keywords diagnostics, expert, stability, vibration

Vibration data is important in determining the condition of rotating machinery. Automation of the diagnostic evaluation of certain simple faults can be easily implemented. The power and speed of modern computer chips and data acquisition systems have opened a new range of capabilities not possible even 5 years ago. The mass-produced items are the most likely candidates for small expert systems. In the automotive industry, driverviewable screens are common, and more technical diagnostics of a power plant's performance are available to trained mechanics with access to the proper equipment for connecting to the

Received 20 May 2001; accepted 18 October 2001.

Address correspondence to R. Gordon Kirk, Department of Mechanical Engineering, Randalph 0238, Virginia Polytechnic Institute, Blacksburg, VA 24061, USA. E-mail: gokirk@vt.edu onboard data-acquisition system. While it is possible for each user installation to develop a unique expert system, that is highly inefficient and, in general, undesirable.

The advances in computer languages in recent years have made it difficult to keep pace with the graphical capabilities available to programmers. The end-users expect the system to have the latest look and feel. Many older technicians and experts may see little need for more advanced capabilities that go beyond the raw vibration signatures, but younger technicians and engineers without the same vast personal experience may not be familiar with the faults of the machinery and may require a more automated system to solve problems.

Over the past 15 years, there has been an explosive growth in the use of expert systems in engineering applications. More than 150 commercial expert systems are currently on the market for engineering-specific tasks. The only areas for which a greater number of expert systems have been developed are the business, medical, and manufacturing fields (Durkin 1996). Many of these systems are meant for use in power plants and for other heavy industrial applications. Most of them (particularly those designed for nuclear power plants) have been designed to aid plant personnel in sorting through and interpreting the assorted alarms and warnings the plant equipment generates (Corsberg 1987; Vale and Mows 1993). Few, however, have been designed to be low-cost systems for smaller power plants, capable of reading data from standard plant sensors and creating its own set of alarms and bringing them to the attention of the operator.

Major efforts were made at the Virginia Polytechnic Institute (Virginia Tech) Rotor Dynamics Laboratory to develop an inexpensive system to monitor, evaluate, and diagnose rotating machinery in industrial applications such as power plants through the use of a personal digital computer. That research began with the development of an off-line rule-based expert system (Kirk et al. 1989a, 1989b; Typrin et al. 1992). The extension of this off-line system into an on-line expert system capable of monitoring multiple machines with trending and diagnostic capabilities was the basis of additional work at Virginia Tech (Hoglund 1989; Kirk et al. 1991; Pawtowski 1996; Typrin 1992). That research 
was similar to the VARMINT system that was developed at Design Maintenance Systems in North Vancouver, BC (Liddle et al. 1993).

A common feature of many expert systems is the use of a hard-wired knowledge base that was probably developed on the basis of experience and is capable of detecting known problems having known systems. Others systems have the ability to learn from the system under evaluation and add to the existing knowledge base in some fashion. It is now common for automobiles to detect low water, low oil, open or not fully closed doors, and improper lighting elements. The level of sophistication continues to increase in many areas of mass-produced items with cost structures able to support the computer logic essential for detecting and displaying the results of the system analysis. Similar dedicated systems will soon be developed for all critical-path rotating machinery.

\section{EXAMPLES OF FAULTS OF INDUSTRIAL MACHINERY}

It is essential to have knowledge of many known machinery faults to be able to diagnose a new problem in a different, but perhaps similar, piece of rotating machinery. In addition to 15 years in academics and in consulting for machinery analysis and problem solving, the author's (RGK) own personal industrial experience consists of 3 years in aircraft engine development work and another 10 years in working with industrial turbomachinery, including centrifugal compressors, steam turbines, axial compressors, screw compressors, power turbines, hot gas expanders, and centrifugal pumps. A large portion of this experience involved problems with high-pressure centrifugal compressors because of the attention given these machines as a result of the lost value of product in the event of an unscheduled shutdown. Gas pipelines must stay in service all winter in most regions of the United States and United Kingdom, for example. High penalties are paid if the proper volume of gas is not delivered. Many problems with compressors are related to bearings and seals. Others are caused by balance shift due to impeller fits, improper installation of couplings, misalignment, poor foundations, entrapped liquids, incipient surge, rotating stall, pressure pulsation reflection in pipe sections, light rubs, seal rubs, poor lubrication to bearings, and so forth. Each problem must be described to distinguish the vibration signatures and phase relationships or other measurable parameters from other possible problems.

Figure 1 shows a classic case of oil seal reexcitation of a machine at its first critical speed. This condition is also known as shaft whip. The instability frequency locks onto the shaft's first critical speed, and the level of subsynchronous vibration climbs quickly in many cases, causing the machine to trip on high vibration. In this case, the oil seals are essentially locked and are acting as 360-degree bearings to drive the instability. The remedy is to modify the seals and soft-start the compressor so as to center the oil seal rings (Kirk, 1986). The resulting stable machine frequency spectrum is shown in Figure 2.

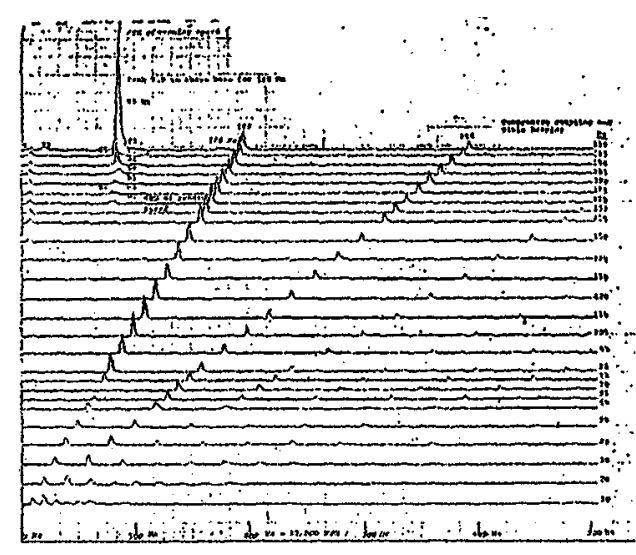

FIGURE 1

Oil seal-excited shaft whip instability in a centrifugal compressor.

A similar spectrum can result from the driving mechanism of a gas labyrinth seal or from aerodynamic excitation caused by pressure distortion in a high-pressure compressor.

If the oil seals are not locked but are freely floating, a different spectrum of low-ratio subsynchronous vibration that tracks running speed results. A classic example is shown in Figure 3. In this case (Kirk and Simpson 1985), the problem was a warped seating endplate for the oil seal ring-lapped radial sealing surface. This caused the seal-lapped surface to be destroyed in a short time. The frequency spectrum, with the seal seating corrected, is given in Figure 4.

At high speed and full pressure, with live gas, the same compressor as that referred to in Figures 3 and 4 shows the shaft whip instability, as shown in Figure 5. In this case, the excitation mechanism is not the oil seals, but the gas labyrinth seal of the balance drum. The fix was to provide a purge flow at the balance drum's entry so as to block and prevent the forwardswirling flow from last-stage impeller leakage to the balance drum.

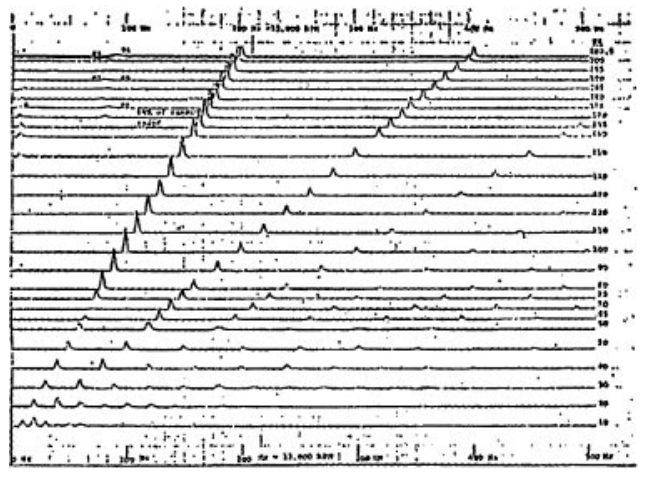

FIGURE 2

Elimination of oil seal-excited shaft whip instability in a centrifugal compressor. 
EXPERT SYSTEMS

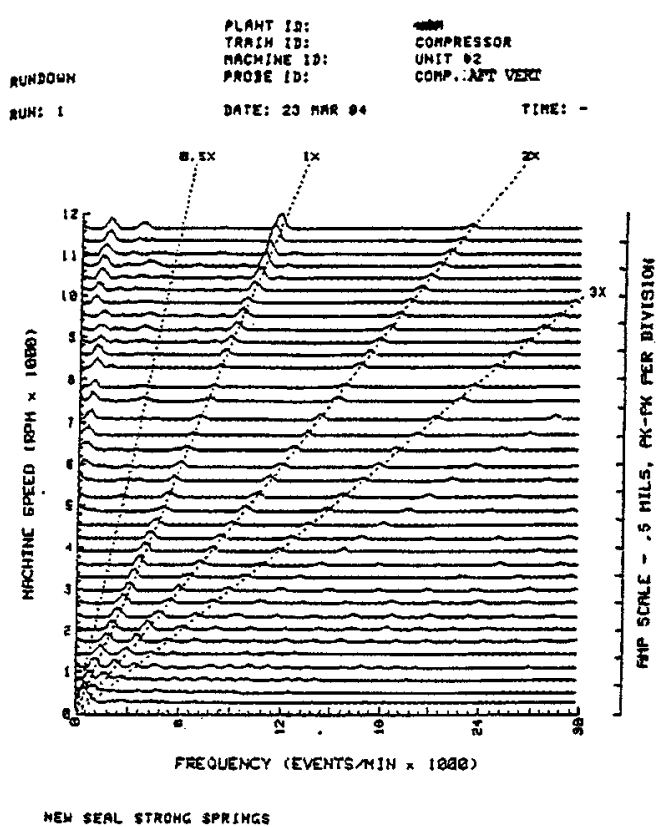

FIGURE 3

Oil seal ring instability driving compressor vibration at low order of rotor speed after $5000 \mathrm{rpm}$.

Other examples of machinery faults in actual shop or field operations of full-scale machinery have been published (Kirk 2000). The problem with fault identification based on limited data is illustrated by Figures 1 through 5. Similar spectra have been observed in numerous other pieces of machinery but result

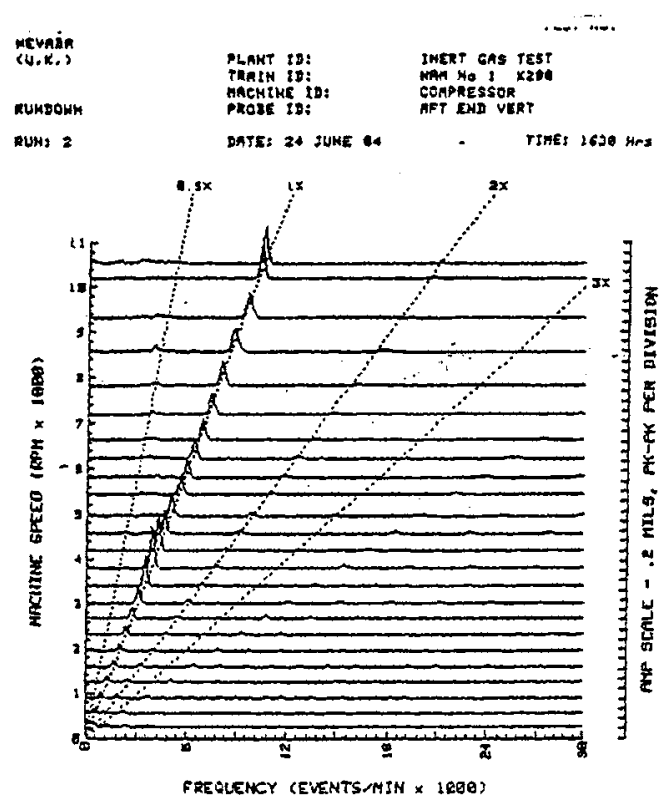

FIGURE 4

Spectrum after seating of oil seal ring and elimination of instability.

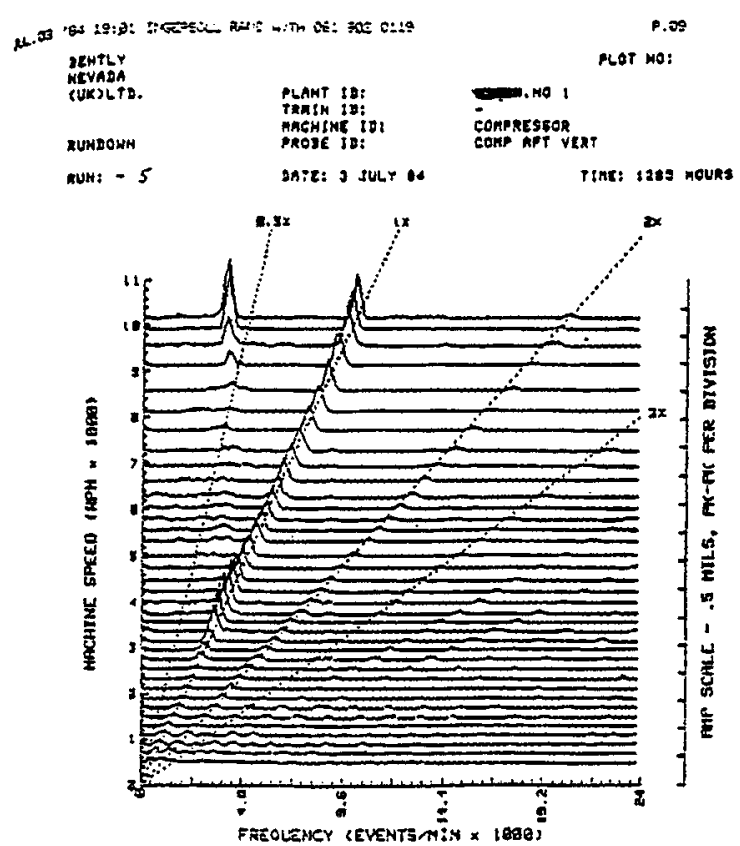

(a)

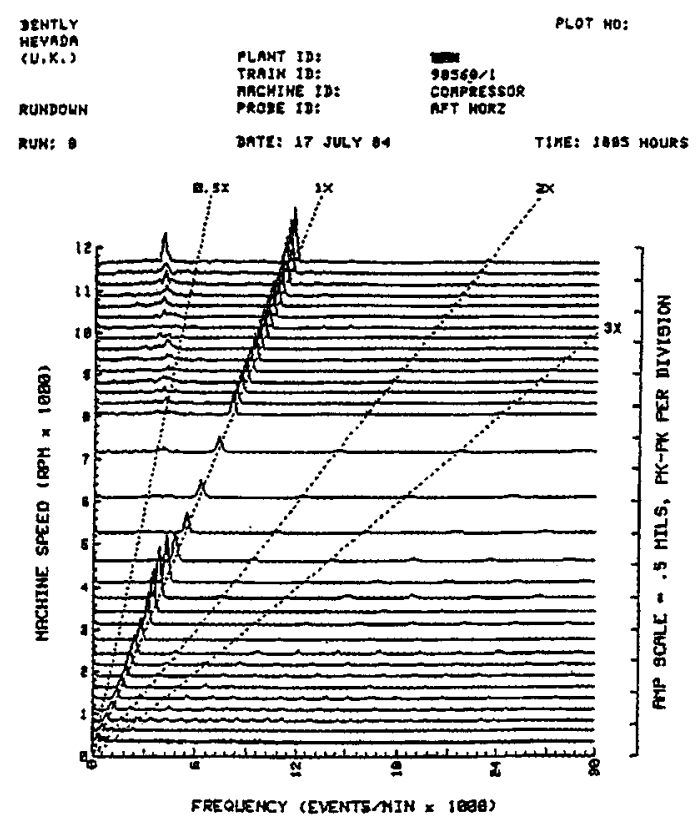

(b)

\section{FIGURE 5}

(a) Shaft whip instability caused by balance drum labyrinth. (b) Reduced shaft whip instability with balance drum labyrinth radial shunt line.

from different causes. The expert must be capable of deciding which mechanism is causingthe instability. Many problems are specific to a particular class of machinery or to machinery with certain components. Only if full details are known can an accurate prediction of cause be made with confidence. 
TABLE 1

Possible Problems in a Centrifugal Compressor

\begin{tabular}{ll}
\hline Seal oil excitation & Insert bearing oil whirl \\
Heavy rub & Thrust-bearing damage \\
Seal oil whirl & Loose rotor component \\
Seal whirl & Rotor critical \\
Shaft whip & Coupling critical \\
Light rub & Overhang critical \\
Loose bearing shell & Electrical excitation \\
Unbalance & Ground loop in instrumentation \\
Electrical or mechanical & Torsional resonance \\
$\quad$ glitch & \\
Misalignment & Process-related problem \\
Bowed rotor & Gear problem \\
Axial rotor rub & Labyrinth seal excitation \\
\hline
\end{tabular}

\section{EXAMPLE OF EXPERT SYSTEM INFORMATION}

For a problem to be diagnosed, it is essential to know the signature, or characteristics, of the problem. It is also desirable that the characteristics be unique to the given problem. This requires that a human expert know a vast amount of information or that such information exist in a retrievable data structure in a computer system. A major hindrance to generation of such a knowledge base is the possible perception by the human expert working in industry that putting all of his knowledge into a computer database would reduce his own usefulness to his company. While this may be true in some cases, the majority of problems requiring a human expert have not been solved previously and, hence, are not in any computer knowledge base. Table 1 lists possible problems in a compressor.

Tables 2 through 4 provide lists of conditions that can be used to determine whether one of these particular rules is a likely to be the cause of a current problem. Lists of problems and conditions in a steam turbine are shown in Tables 5 and 6 . The corrective action for misalignment is given in Table 7 .

Table 8 illustrates the desirable and necessary option for a knowledge base editor. A knowledge base must have an editor to update and add new information to allow the latest known vibration characteristics to be available in the expert system. The neural network of this system is the human expert.

\section{TABLE 2}

Conditions for Unbalance

Rule: Unbalance

The machine does not have subsynchronous whirl.

It has strong one-per-revolution vibration.

It is actually running.

The shaft vibration is dominant, that is, stronger than that of the casing or bearing cap.

The amplitude increases uniformly with speed.

The amplitude is not influenced by a change in operating conditions.

TABLE 3

Conditions for Shaft Whip

Rule: Shaft whip
The machine operates above first critical.
It has a flexible shaft.
It has tight clearance bearings.
It has an operating speed greater than $2.0 \times \mathrm{Ncr}$.
It has nonsynchronous whirl.

TABLE 4

Conditions for Labyrinth Seal Excitation

Rule: Labyrinth seal excitation

The machine operates above the first critical frequency.

There is no shunt line on the balance piston.

The vibration is dominant subsynchronous at the first critical frequency.

The compressor has a high-pressure drop across the balance piston.

The machine has tilting-pad bearings.

The unit has no problems at reduced loading.

An increase in was the source of the vibration increase

TABLE 5

Possible Problems in a Steam Turbine

Rotor unbalance

Loss of material

Rub (on-line/at speed)

Seal rub (on rollup or rolldown) Steam pressure loading

Bad (fluid-film) bearing

Soft foot condition

Rotor bow (on-line bow or rub)

Resonant pedestal

Coupling unbalance

Bent rotor/excessive runout

Misalignment
Slipped coupling
Instability
Steam pressure loading
Cracked rotor
Operating near a critical
Overhang critical
Poor (lost) insulation on inlet
Electrical glitch or scratch
$\quad$ under probe

TABLE 6

Conditions for Misalignment in a Steam Turbine

Rule: Misalignment

The machine is a turbine.

The $1 \times$ vibration changes with loading or temperature.

The vibration characteristics are generally repeatable.

The bearing temperatures are in the normal range for this machine.

A precision level check across adjacent bearings shows a large change with load.

The pedestal axial vibration is greater than $40 \%$ of the radial vibration.

The machine has occasionally had what appeared to be stability problems. 
TABLE 7

Corrective Action for Misalignment in a Steam Turbine

Rule: Misalignment

Limit unit loading to where vibration is acceptable

(for the short term).

Measure hot-to-cold alignment change and then realign unit.

\section{EVALUATION LOGIC}

Theexistence of a knowledge base is necessary but not sufficient for ranking the possible causes of a particular problem. It is essential to rank each possible cause; the resulting confidence factor for each rule is then used to rank the most likely causes. The work at the Virginia Tech Rotor Lab adopted a three-phase logic in which a condition is true, false, or unknown. The equation used in a prolog expert program is given by Hoglund (1989). Each rule requires an original confidence factor, which is part of the knowledge base, and each evaluation of a problem generates a modified confidence factor for each rule. The results of applying such logic during a typical session are shown in Table 9. It is necessary to have an original confidence factor for each rule. The result of the evaluation of all information then produces a modified original confidence factor (MOCF).

For automated expert systems, the response must be determined to be true, false, or unknown by further computer logic. The Virginia Tech on-line system used automated standard evaluation to determine whether vibration levels were low, normal, or excessive. Other features are documented by Liddle and colleagues (1993).

\section{AUTOMATION OF DIAGNOSTICS}

The on-line automation of an expert system similar to that just described would seem to be a simple task. First, some questions must be answered by the control room operator of a given machine. Then the data are collected from a connected data

\section{TABLE 8}

Key Options Required for a Knowledge Base

\begin{tabular}{ll}
\hline \multicolumn{2}{l}{ Rule Menu } \\
F1 Create a new database \\
F2 Views all rules in the database \\
F3 Add a new rule to the database \\
F4 Choose an existing rule to edit \\
F5 Choose a database to edit \\
F6 Exit the Editor program \\
F7 Display remaining RAM memory \\
What part of this rule would you like to edit? \\
F1 Conditions \\
F2 Suggestions for corrective procedures \\
F3 Related standards \\
F4 References \\
F6 Exit to previous menu (Rule Menu) \\
\hline
\end{tabular}

TABLE 9

Modified Original Confidence Factors

\begin{tabular}{lc}
\hline \multicolumn{1}{c}{ Problem } & MOCF \\
\hline I have concluded that your machine's difficulty & \\
may be due to: & \\
Seal whirl & 0.90 \\
Insert-bearing oil whirl & 0.90 \\
Seal-oil whirl & 0.89 \\
Seal-oil excitation & 0.87 \\
Axial rotor rub & 0.85 \\
Shaft whip & 0.81 \\
\hline
\end{tabular}

acquisition system and the answers obtained. For a specific machine, this could be simple. If the on-line expert system is to operate for all machine systems, it is necessary to configure the data acquisition in a simple manner, so a well-written program is required. In 1989, specific instruments had to be purchase for each function needed, for example, a Bently Nevada Corp Digital Vector Filter (BNC DVF) to pull data concerning the amplitude and phase of shaft probes, and the spectrum required a specific box to collect and obtain the frequency content. By 1994, all these instruments were virtual monitors but were still programmed. Today, it is possible to use a laptop to collect data via available hardware and MATLAB to compute the spectrum, response, and phase.

\section{Desirable Features}

The desirable features of an on-line expert diagnostic system are as follows:

The ability to run in off-line mode if no machine data is available The ability to update the rule condition list

The ability to add new rules

The ability to recommend solutions

The ability to refer to publications concerning the problem

The ability to refer to standards relating to the problem

The ability to compute spectrum content and response and phase by using standard calculations

The ability to allow initial setup to be retained in the control room of the computer system

The ability to hold files of machine specifications

The ability of the operating system and graphics to be compatible with state-of-the-art languages

\section{EXAMPLE OF APPLICATION: OFF-LINE EXPERT SYSTEM}

A recent and, in fact, the first application of the off-line expert from Virginia Tech to a real problem with an unknown cause will be discussed briefly to further illustrate the use and application of such an expert system. The power plant at Virginia Tech has a 7-megawatt steam-turbine-driven generator that operates to provide power for peak and normal loading from the student dorms and, in addition, supplies power to a small part of the town of Blacksburg. The 15-year history of the turbogenerator 
includes problems with misalignment resulting in bearing instability due to the unloading of the close coupled inboard bearings. The original journal bearings were converted to a pressure dam design to help reduce this instability problem. The system had been running with acceptable vibration for 6 years. In the summer of 2000 , the turbine was upgraded to produce a more dependable and efficient driver. The new turbine rotor plus new bearings and seals for both the turbine and generator were being installed in early autumn, and start-up was needed prior to cold weather. When the rotor was to be installed, it was discovered that an original seal fix had not been documented, so the rotor could not be installed. The OEM had modified seal parts on site in 2 days, and thus this potentially major problem with the $\$ 1.2$ million re-rate was averted. The rotor was installed, aligned, and slow-rolled.

The start-up was then apparently of no concern and no instrumentation or help was requested of the Virginia Tech Rotor Lab staff. Previously, BNC DAI 108 data collection had been used when the bearing instability problems were causing near trips some 6 years earlier. In addition, the on-line expert system was installed and developed at Virginia Tech just after that, but since the problems had been solved, the final system was never upgraded and put into service; the data collection boards are still installed behind the control panel and the lines still run to the instrumentation area where the computer monitoring was to be conducted. The turbine was down each summer, which made verification of the on-line system more difficult. The final on-line system was never installed - completion was delayed by numerous personnel problems and funding problems - and the final computer system was never installed at the power plant.

On the Monday following the first attempted start of the new turbine on the previous weekend, a phone call was received from the power plant's instrumentation engineer. They had not been able to get the unit to function because the vibration was excessive-above the warning level and near trip. The initial suspicion was high subsynchronous vibration due to misalignment, suspicion was or maybe a rub in the turbine where the retrofit seals had been installed.

The first visit to the power plant, less than 25-min walk from the Mechanical Engineering Department, revealed that the vibration, in the turbine was actually very low. It was in the generator that the vibration was high, especially in the outboard probes. The generator bearing caps and turbine foundation had excessive vibration that was evident even without taking readings but that was confirmed by hand-held velocity probe readings taken using the small BNC TK-83 hand-held field-balance monitor. The concrete block foundation under the generator had in excess of 1 mil of vibration at running speed. The generator bearing caps were covered at that time, so initial confirmation of the bearing caps levels was not made.

Concerns shifted to potential causes of the high vibration. Problems with generator windings or other damage from the rerate work were suspected; misalignment and coupling problems were not high probabilities because the turbine was running at
TABLE 10

Results Based on the Rockland Knowledge Base

\begin{tabular}{cc}
\hline Problem & MOCF \\
\hline I have concluded that your machine's difficulty may be due to: & 0.88 \\
Eccentric motor mass (no electrical problem) & 0.83 \\
Unbalance (centerhung machine) & 0.70 \\
Misalignment (parallel) & \\
\hline
\end{tabular}

such a low vibration. A call to the engineering department of the generator's manufacturer was planned by the power plant manager, if it could be arranged.

The use of the off-line expert system was tried first, using a general knowledge base for machinery that had been developed in the late 1980s based on the Rockland diagnostic table. The summary of possible causes of this problem is shown in Table 10.

The results were directly related to how the questions from the expert system were answered. Observation of this information helps to clarify why a specific cause has been selected. The utility of the ability to include the influences of elements not known allows a greater number of possible problems to be shown by the system. Table 11 displays the query and the user response for this specific session. The results are likely to be of no surprise and

\section{TABLE 11}

Responses of the Rockland Knowledge Base to Results Shown in Table 10

The conditions used to prove eccentric motor mass (no electrical problem) are:

$\mathrm{Y}$ The machine is a motor unit.

$\mathrm{Y}$ The frequency is $1 \times$, i.e., running speed.

$\mathrm{Y}$ It also has vibration at $1 \times$ and $2 \times$ line frequency.

$\mathrm{Y}$ The radial vibration is dominant.

D The vibration is steady.

$\mathrm{Y}$ The vibration envelope is narrowband.

The conditions used to prove unbalance (centerhung machine) are:

$\mathrm{Y}$ This machine is centerhung.

$\mathrm{Y}$ The frequency is $1 \times$, i.e., running speed.

$\mathrm{Y}$ The radial vibration is dominant.

D The vibration is steady.

D The inboard and outboard vibrations are in phase.

$\mathrm{Y}$ The vibration envelops is narrowband.

The conditions used to prove misalignment (parallel) are:

D It has both $1 \times$ and $2 \times$ vibration.

D The vibration is steady.

$\mathrm{Y}$ The radial vibration is dominant.

D The inboard and outboard vibrations are 180 degrees out of phase.

Y The vibration envelope is narrowband.

Y, yes; N, no; D, don't know. 
TABLE 12

Results of the Motor Knowledge Base

\begin{tabular}{lc}
\hline \multicolumn{1}{c}{ Problem } & MOCF \\
\hline $\begin{array}{l}\text { I have concluded that your machine difficulty } \\
\text { may be due to: }\end{array}$ & \\
Seal rub & 0.87 \\
Weak/light foundation & 0.76 \\
Bad (fluid film) bearing & 0.71 \\
Loose bearing/pedestal & 0.68 \\
Loose part & 0.64 \\
Misaligned journal bearing & 0.63 \\
Resonant pedestal/system & 0.62 \\
Loss/gain of material & 0.61 \\
Rotor unbalance & 0.61 \\
\hline
\end{tabular}

are, in fact, similar to what any experienced machinery engineer would place high on the list of possible causes.

Another knowledge base designed specifically for motors had been developed and was tried next. The results of this knowledge base's evaluation are shown in Table 12. Here, more possible causes of the excessive vibration were offered, so the knowledge base was of greater help in resolving the problem.

The motor-specific knowledge base gave one answer not expected by the authors. This could indicate a lack of experience in motor or generator vibration problems, and it precisely demonstrates the importance of having an expert system in place to double-check and, more important, to enhance personal experience and knowledge.

The search also revealed concern for the foundation, the bearings, lost parts, and general rotor unbalance.

These results were generated prior to the second visit to the power plant (Figs. 6 and 7). Another consultant had been called in and had taken data using his data acquisition system. Those results pointed to possible alignment problems and there was also an indication of impacting or contact in a bearing. Upon comparison of the results, a recommendation of inspection for a seal rub on both ends of the generator was agreed upon and

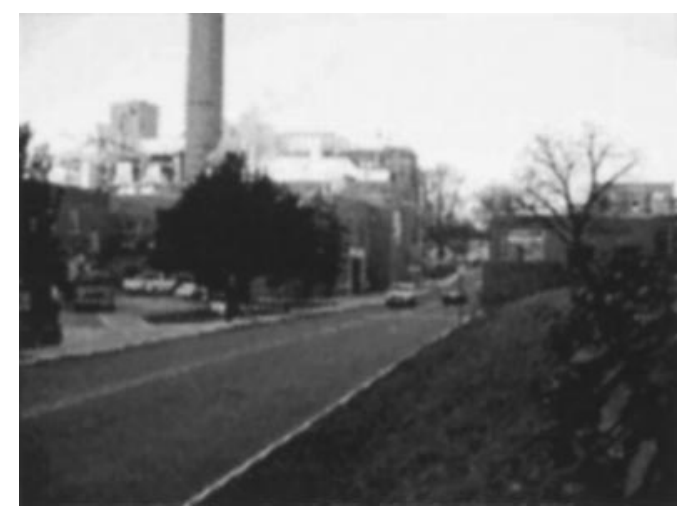

FIGURE 6

The power plant at Virginia Polytechnic Institute.

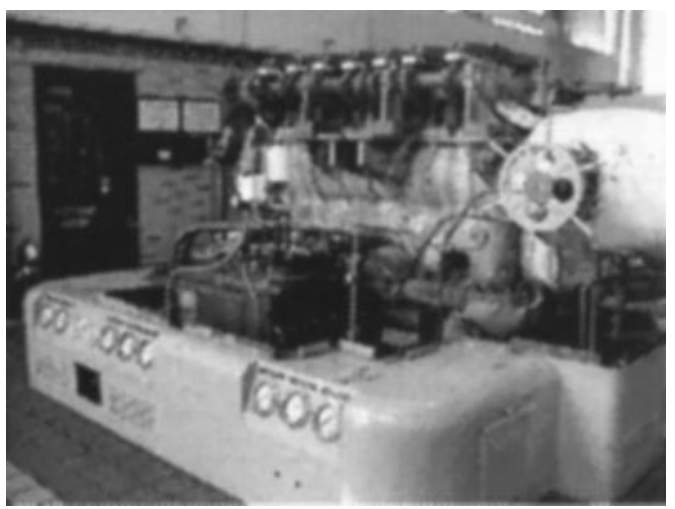

FIGURE 7

A Worthington steam turbine.

accepted by the plant manager. A seal rub was predicted to have the highest probability of being the cause of the problem. The plant manager also insisted on a check of the alignment during the teardown and seal-check work.

The response listing for problem, seal rub, are presented in Table 13. The resolution of a problem is a very complicated process as illustrated by the response list for this problem. At this point, the machine had not been opened and no hard-copy data were available from a start-up or shut-down. The question on the table was Is this a seal rub?

The next day, it was confirmed that a 4- to 6-mil interference had been detected during the seal check in the outboard generator

\section{TABLE 13}

Responses of the Motor Knowledge Base to Results Shown in Table 12

The conditions used to prove Seal rub are:

$\mathrm{Y}$ The $1 \times$ vibration is nearly constant with loading and/or temperature.

$\mathrm{Y}$ There is more $2 \times$ vibration in the motor than usual.

$\mathrm{Y}$ The $1 \times$ vibration on the casing is higher than usual.

D The phase angle either wanders or is cyclical at constant loading.

Y The vibration characteristics are generally repeatable.

D Recent balancing attempts have not been as predicted.

Y The vibration on the motor has changed recently.

$\mathrm{Y}$ The motor vibration is higher than the driven-equipment vibration.

Y An oil sample shows that the oil is relatively clean.

$\mathrm{Y}$ The bearing temperatures are in the normal range.

$\mathrm{Y}$ There is no unusual noise coming from the motor.

$\mathrm{N}$ There is an unusual noise in the bearings.

Y Dial indicator check shows that the shaft is true.

D The vibration phase angle changes opposite to rotation.

Y Seal work has recently been completed.

D The shaft relative vibration waveform appears flattened or nonuniform.

Y, yes; N, no; D, don’t know. 


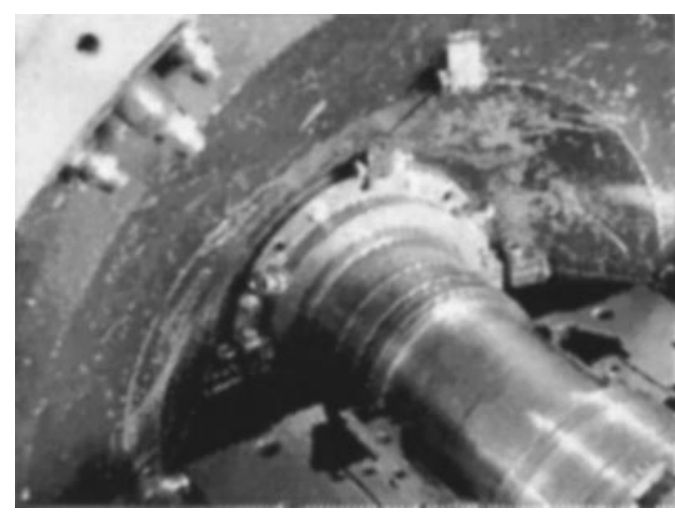

FIGURE 8

Seal rub area at the generator's outboard bearing, showing seal rub marks in Shaft.

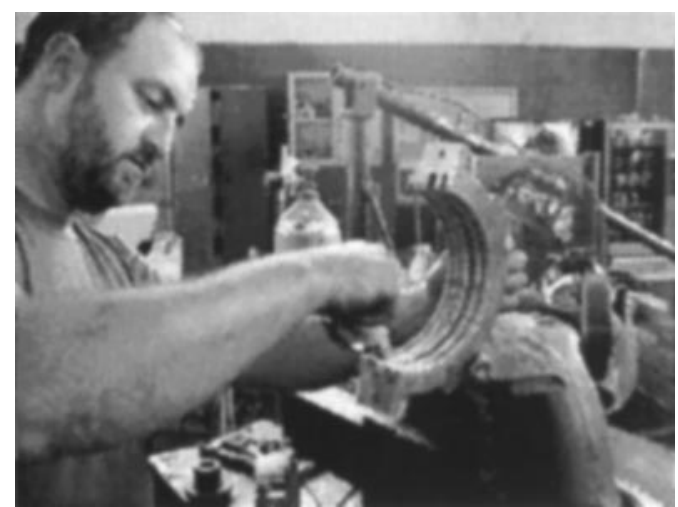

FIGURE 9

Scraping in the seal clearance.

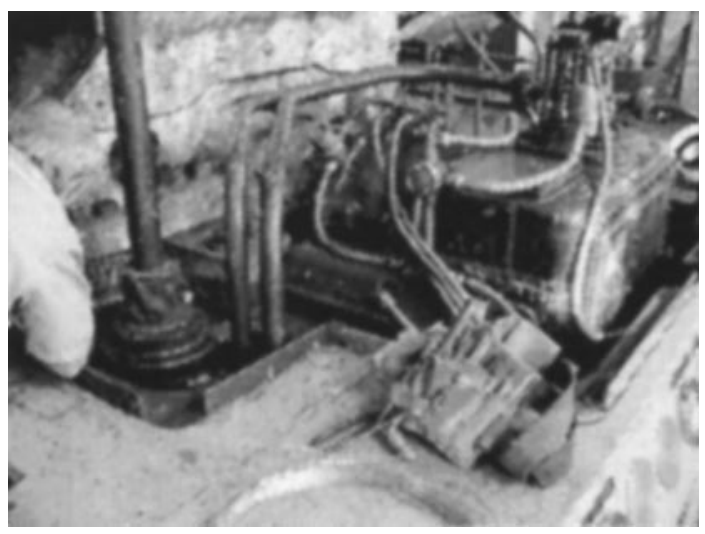

FIGURE 10

Steam end, showing probe location.

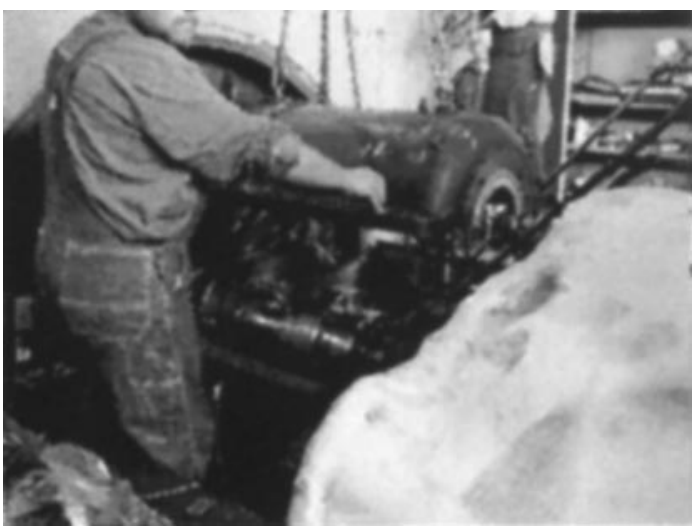

FIGURE 11

Replacing the coupling cover after the fix.

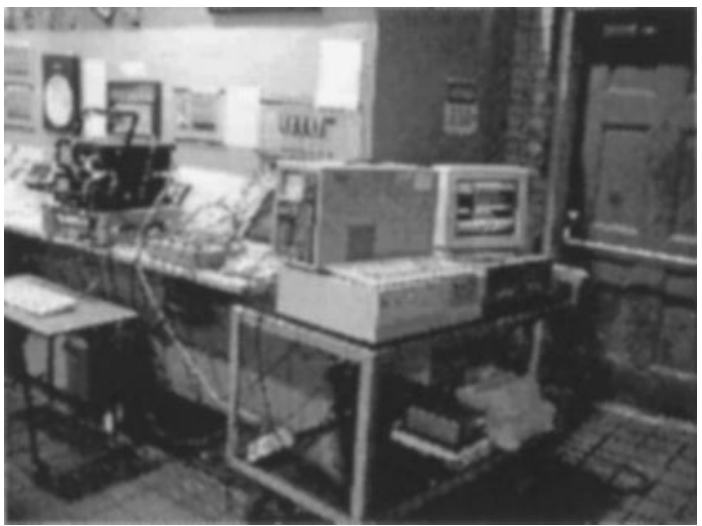

FIGURE 12

DAI-108, FFT, DVF-2, orbit O-scopes, switchbox, ADRE computer, and printer.

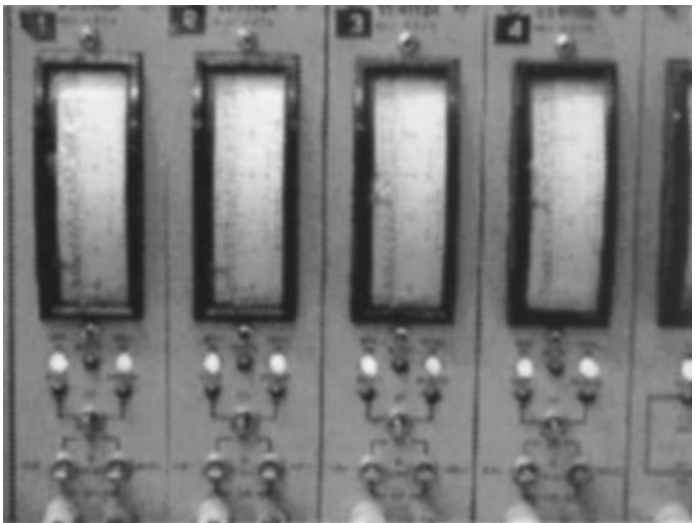

FIGURE 13

Vibration meters after the seal fix. 


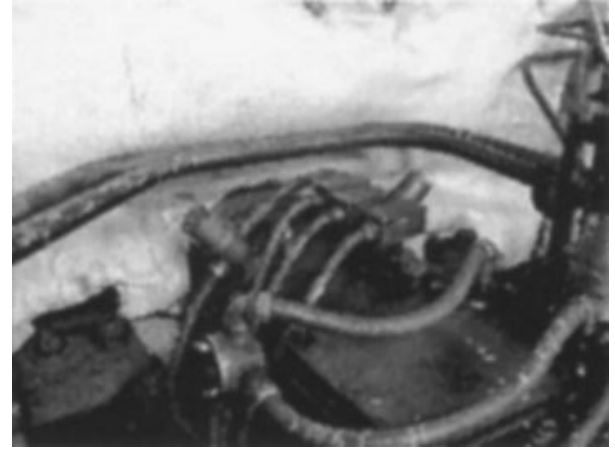

FIGURE 14

Shaft probes on the turbine's outboard.

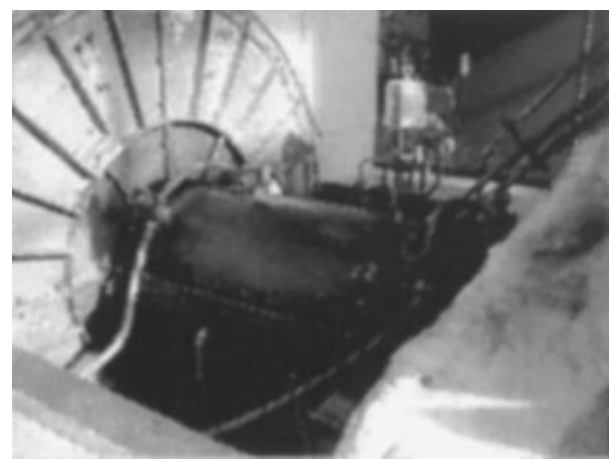

FIGURE 15

Inboard probe locations.

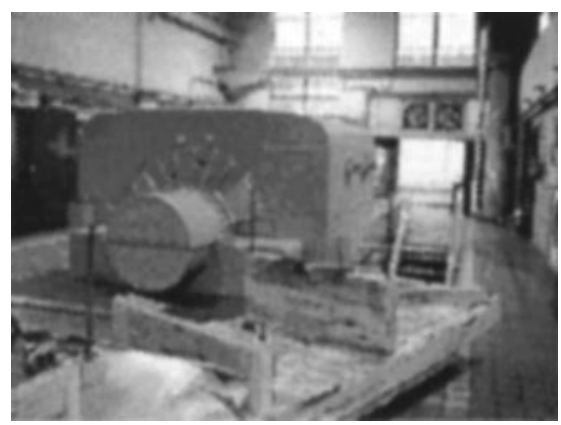

FIGURE 16

Location of outboard generator.

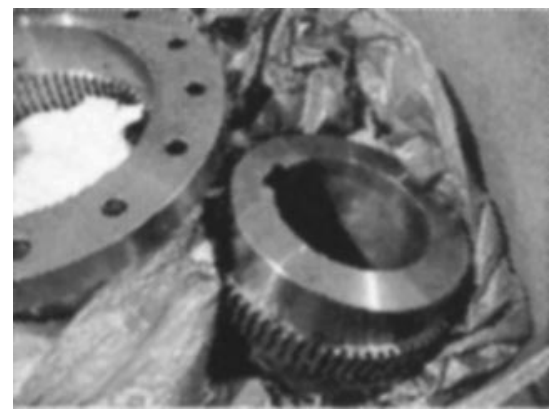

FIGURE 17

Gear coupling hub and sleeve.

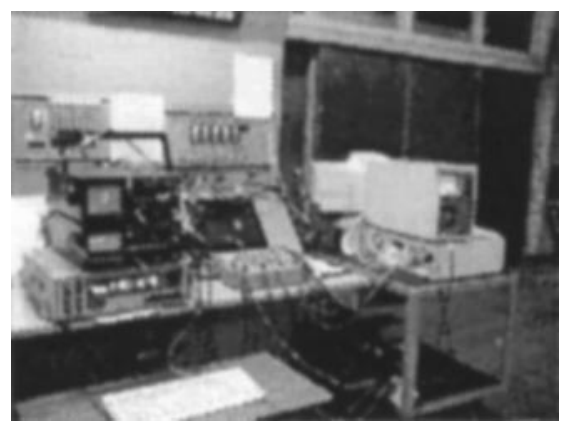

FIGURE 18

Final data-acquisition system for orbits.

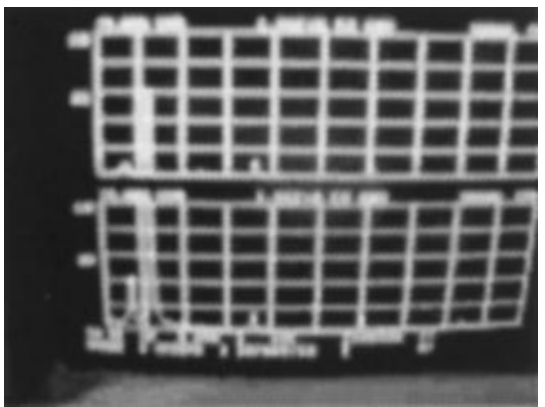

FIGURE 19

Steam whirl peak hold and Instantaneous spectrum.

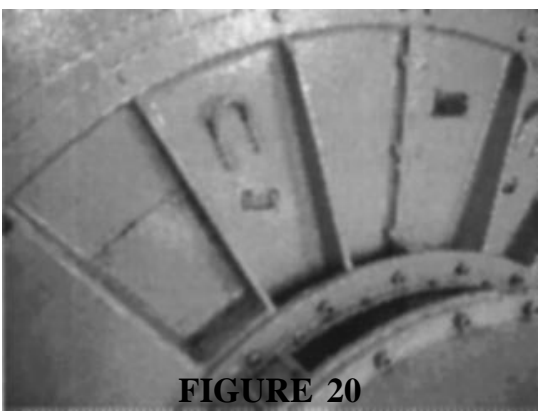

OB generator inspection port.

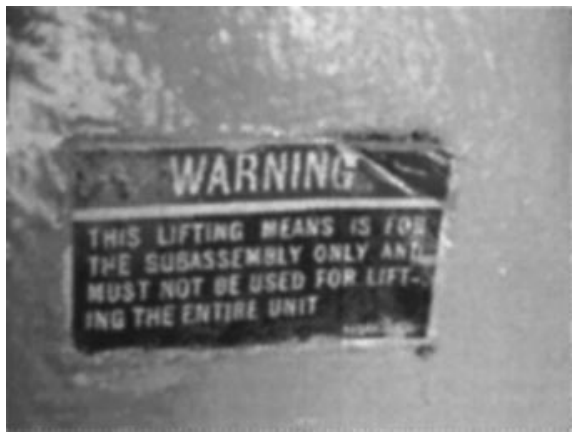

FIGURE 21

Warning sign from generator shown in Figure 20. 


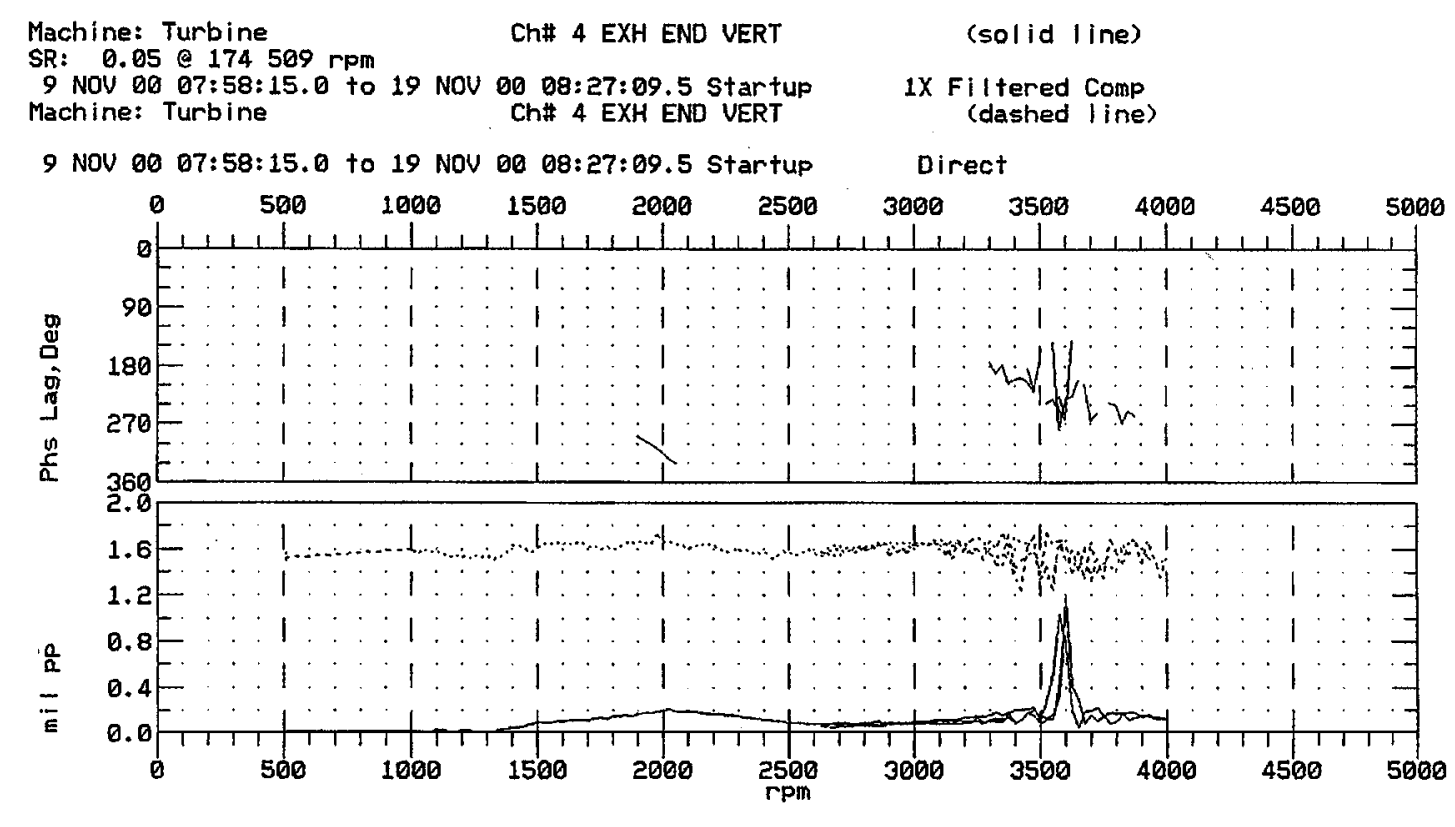

FIGURE 22

Turbine inboard vertical, 11-09-2000.

bearing. The outer seal was scraped to secure the proper clearance (Figs. 8 and 9). The inner air seal was not removed nor was the clearance increased.

The alignment had been checked and was as desired for the hot machine; hence, no change was made to the existing alignment. The machine was readied for start-up (Figs. 10 and 11).

Before start-up, a data collection cart was moved from the rotor lab of Virginia Tech using DAI-108, and cables were run for all eight bearings from the BNC control panel meters, as shown in Figure 12. The set-up was just finished and the first check of the data acquisition system was the start-up. Unfortunately, a high level of 60-cycle ground loop was in the instrumentation, so the at-speed readings were originally in error as they were recorded by the DAI-108. The meters were correctly grounded and isolated. The start-up was recorded using the DAI-108 for documentation of the system's vibration. The meters were all

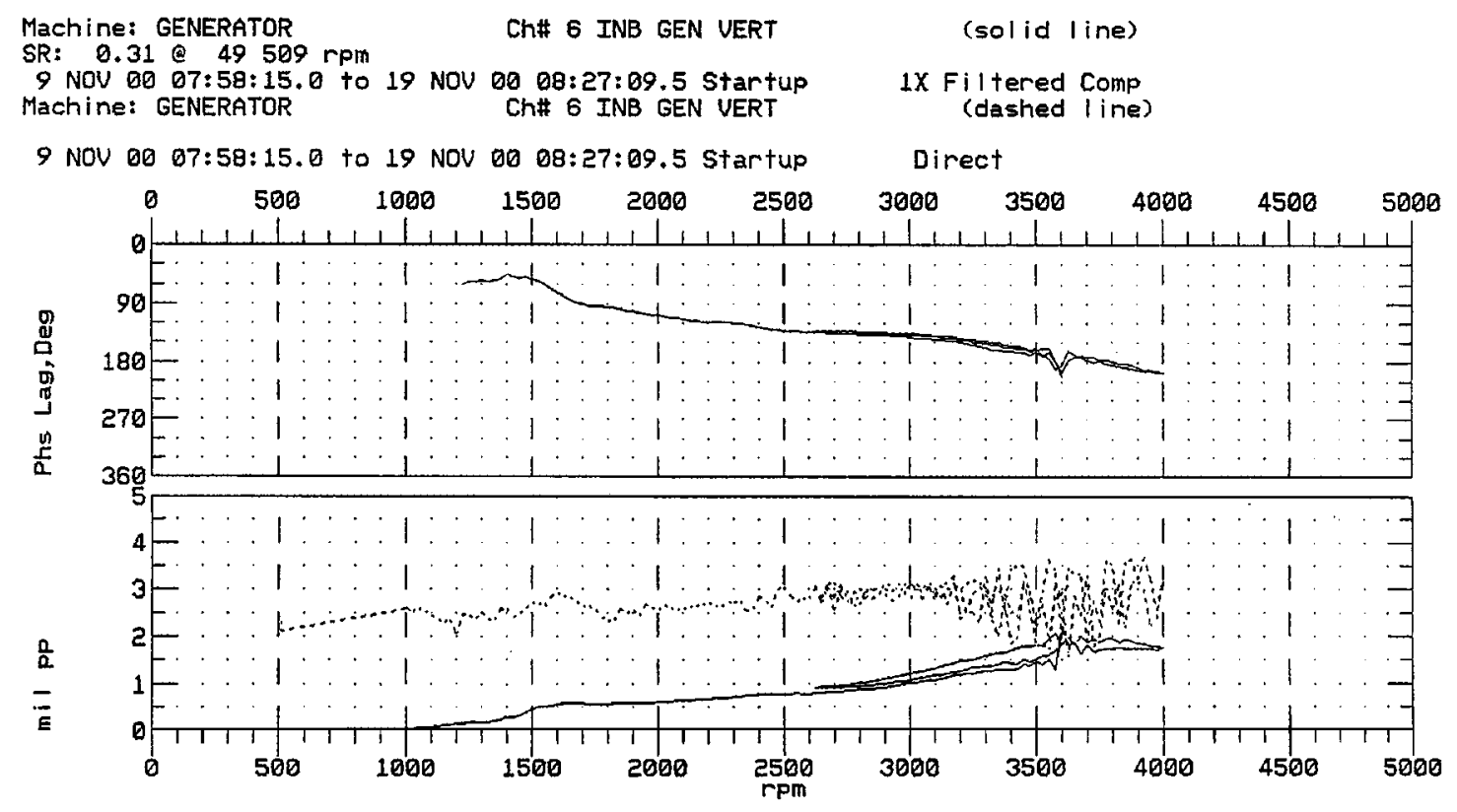

FIGURE 23

Generator inboard vertical, 11-09-2000. 


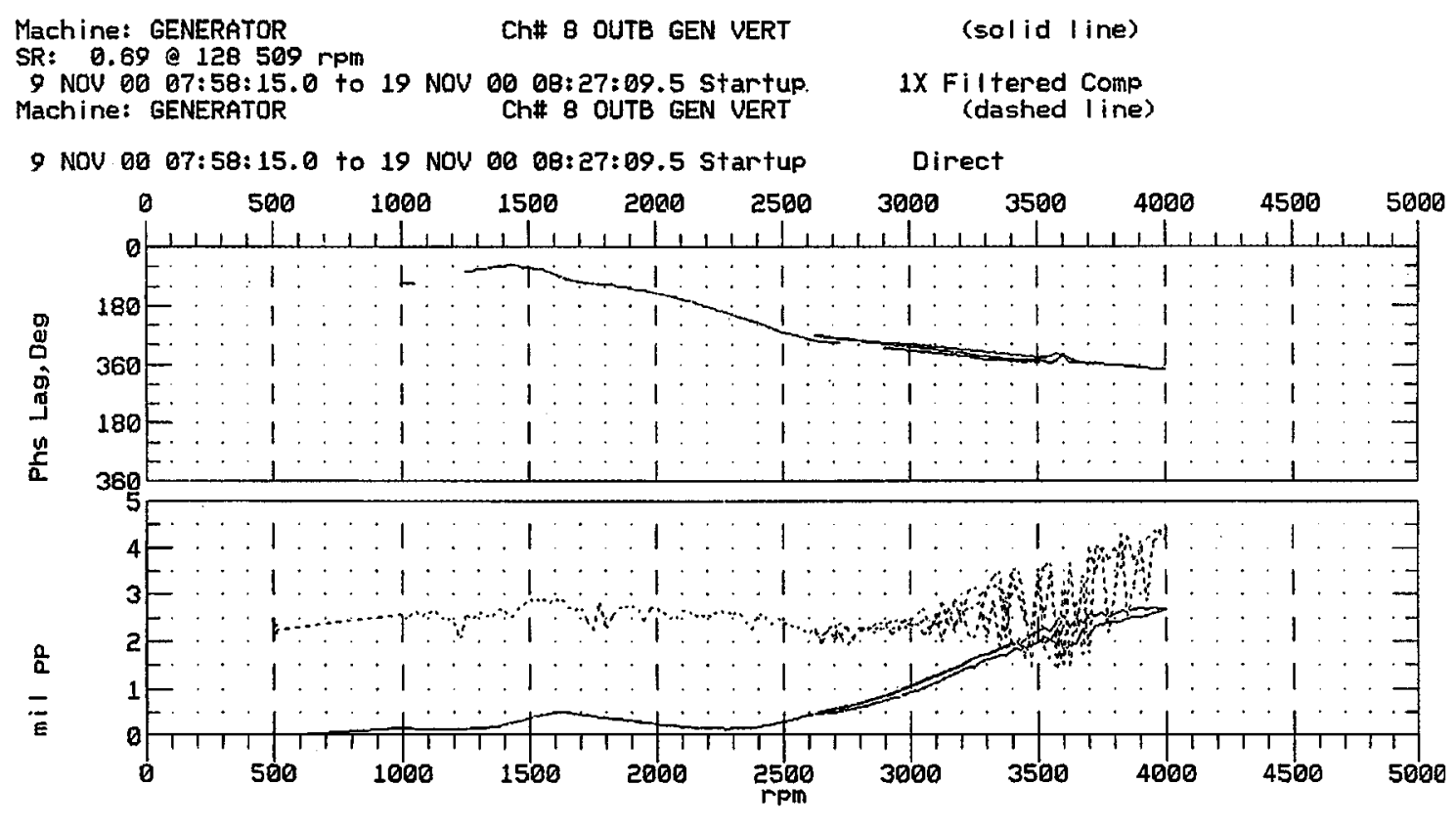

FIGURE 24

Generator outboard vertical, 11-09-2000.

below the warning levels at that time (Fig. 13), but the highest was the generator's inboard probes, so the mechanics were wishing the inboard bearing seals had also been scraped. The levels were close to the warning setting, but the actual vibration was lower because the meters overall were taking into account the scratches on the shaft.
The probe locations are shown in Figures 14, 15, and 16. The outboard generator probes were under the enclosure on that end and the shaft was not easy to see, nor was it easy to take stick readings. The gear-coupling hub and flange that were changed are shown in Figure 17. Continuous running at full power was monitored using instrumentation as shown in Figure 18; it is

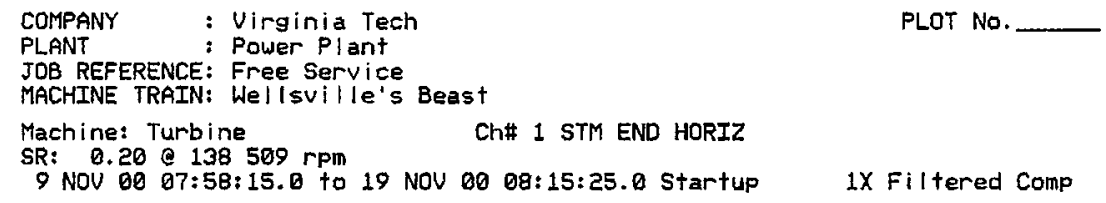

0.20 mil pp Full Scale

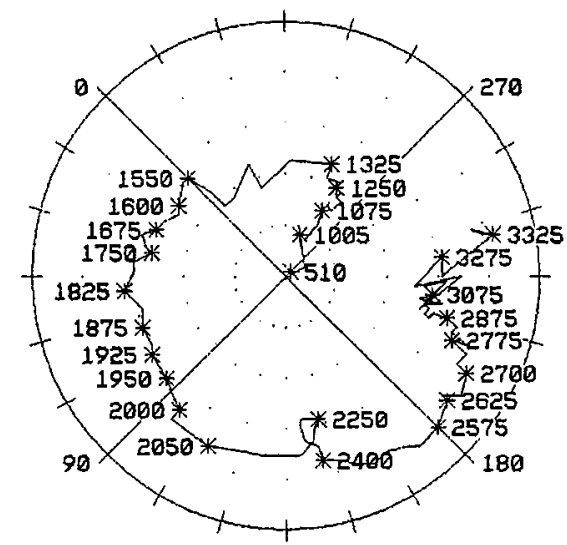

FIGURE 25

Turbine outboard bearing, horizontal polar plot. 

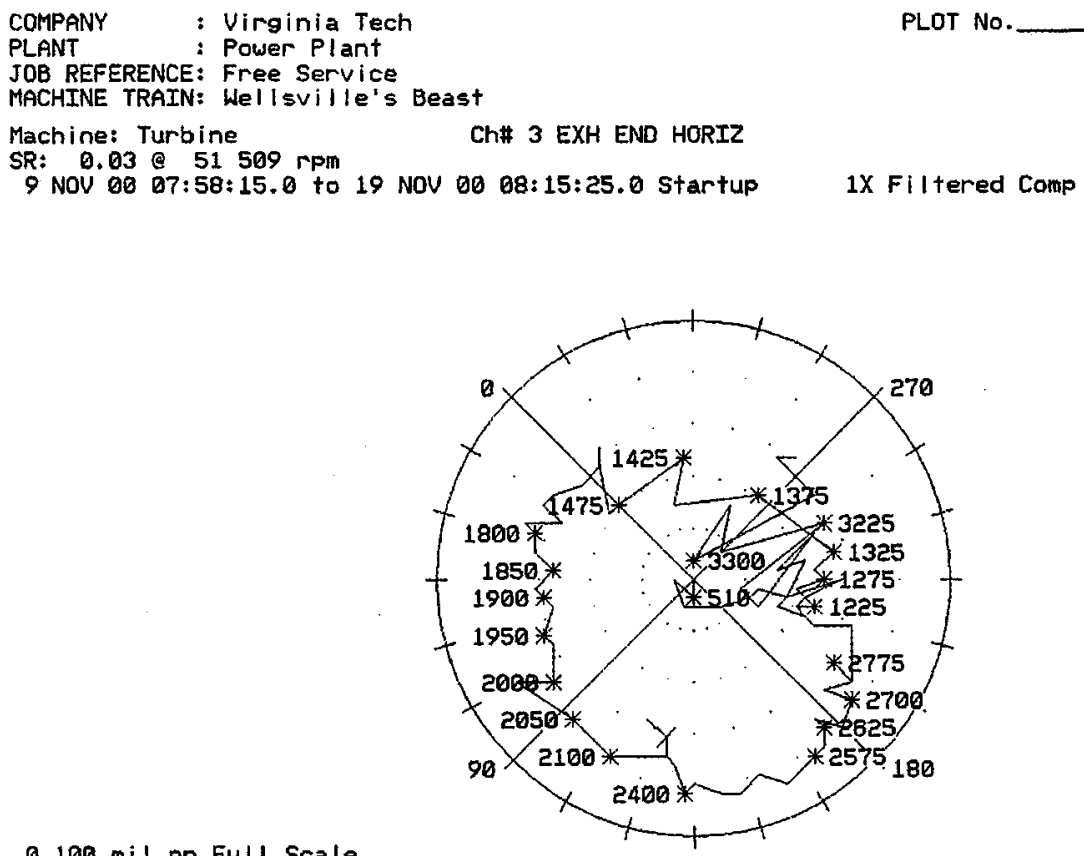

FIGURE 26

Turbine inboard bearing, horizontal polar plot.
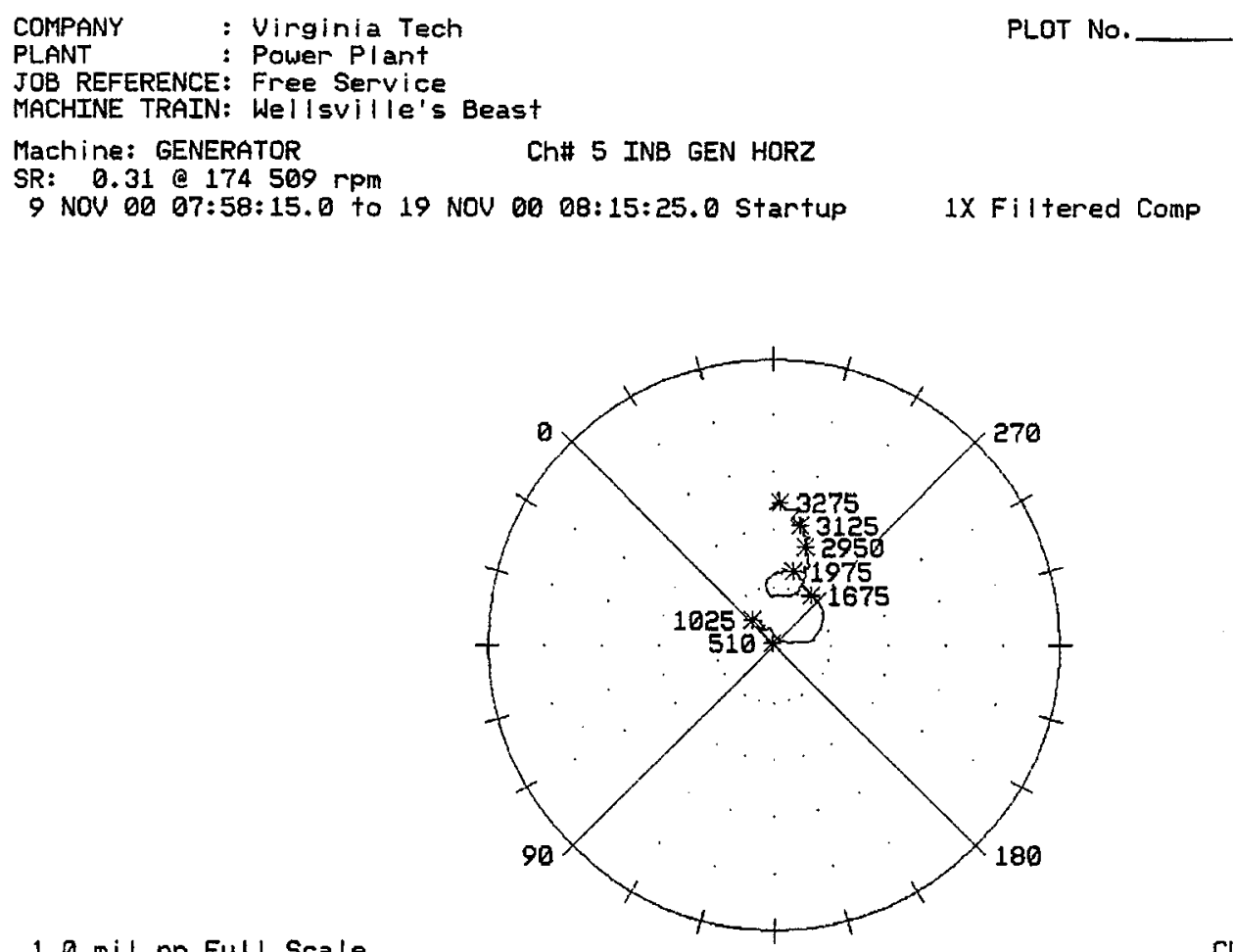

FIGURE 27

Generator inboard bearing, horizontal polar plot. 


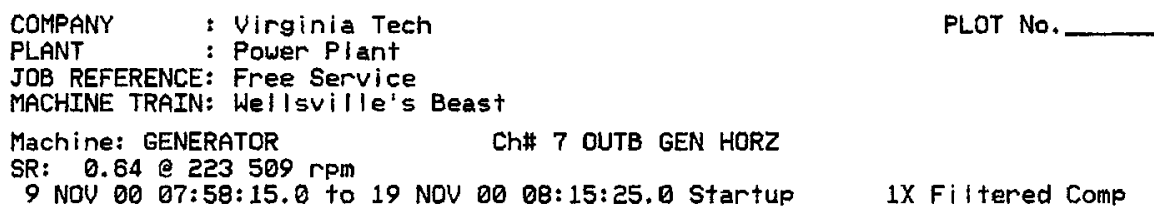

$1.0 \mathrm{mil}$ pp Full Scale

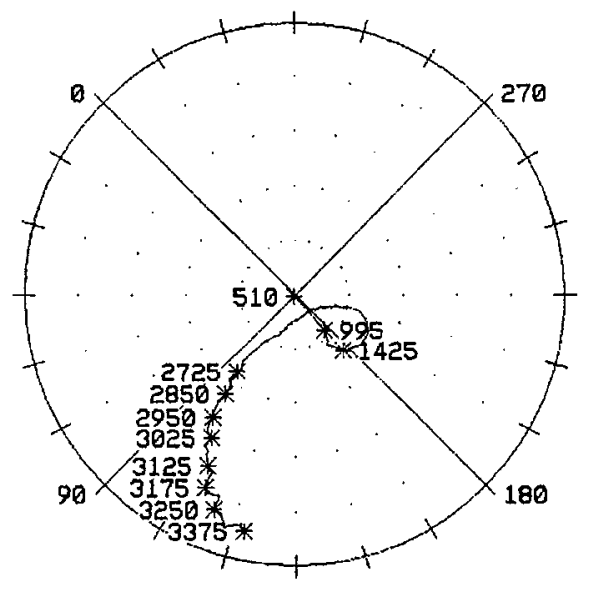

FIGURE 28

Generator outboard bearing, horizontal polar plot.

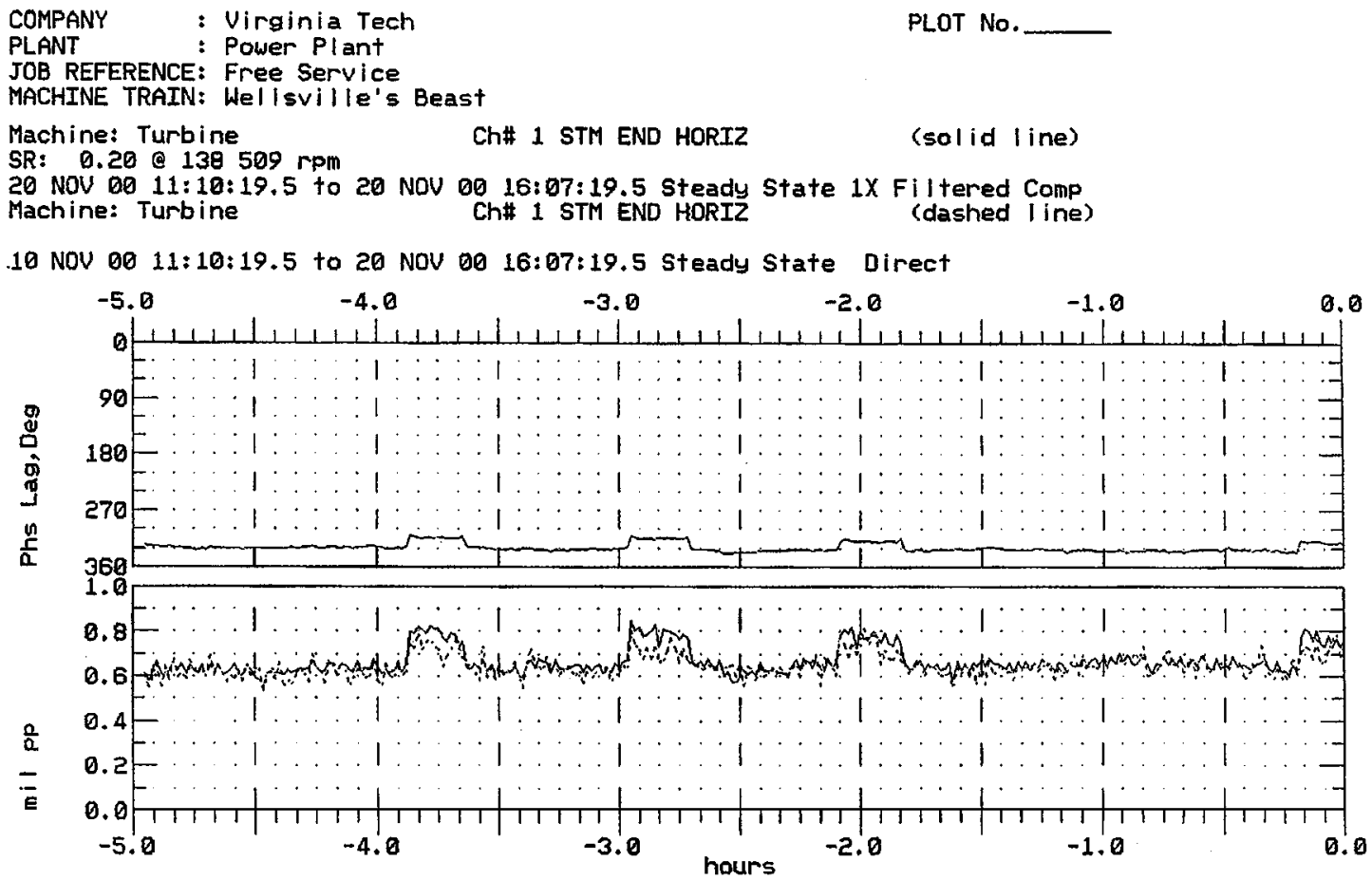

FIGURE 29

Turbine trend plot, outboard horizontal. 


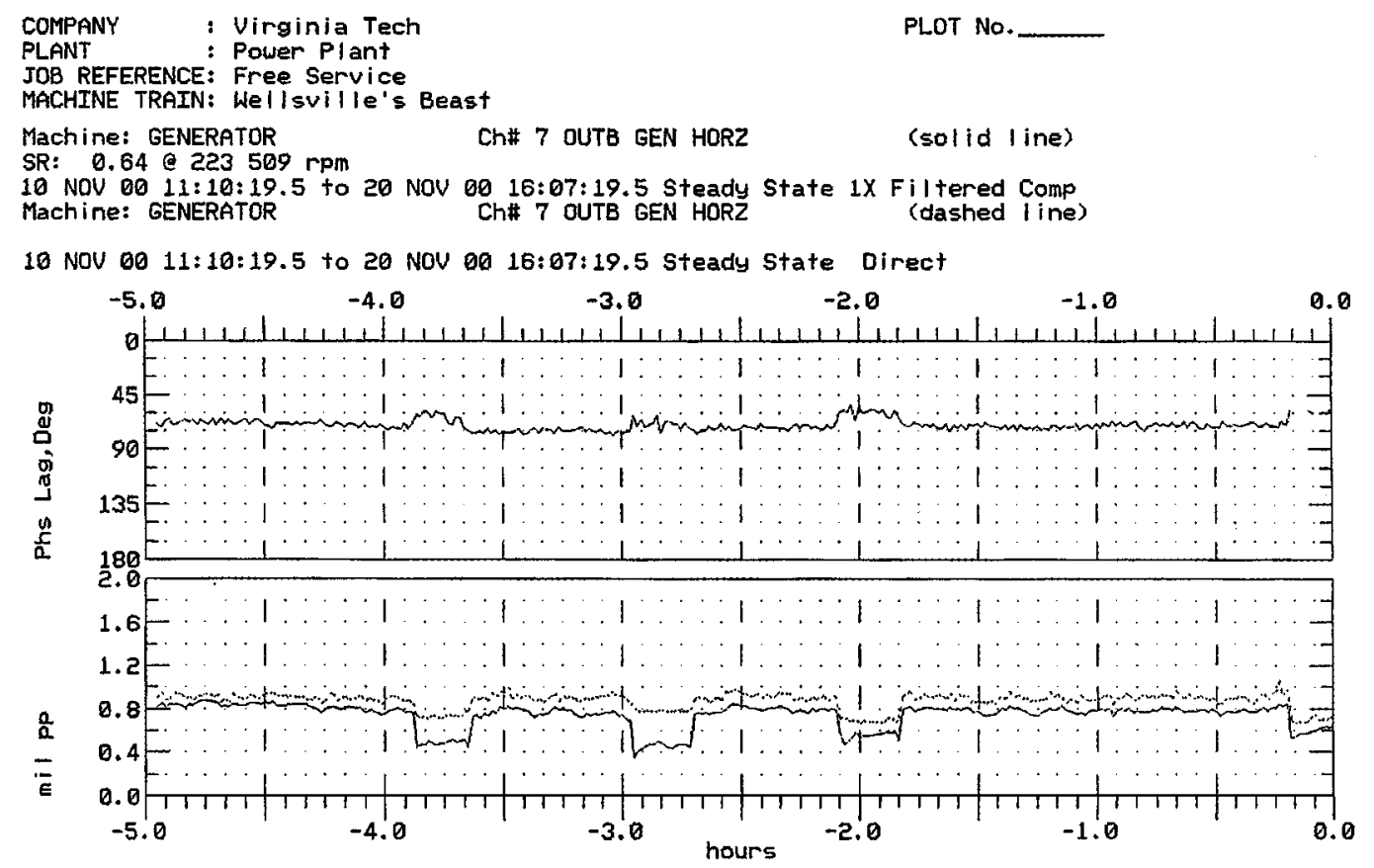

FIGURE 30

Generator trend plot, outboard horizontal.

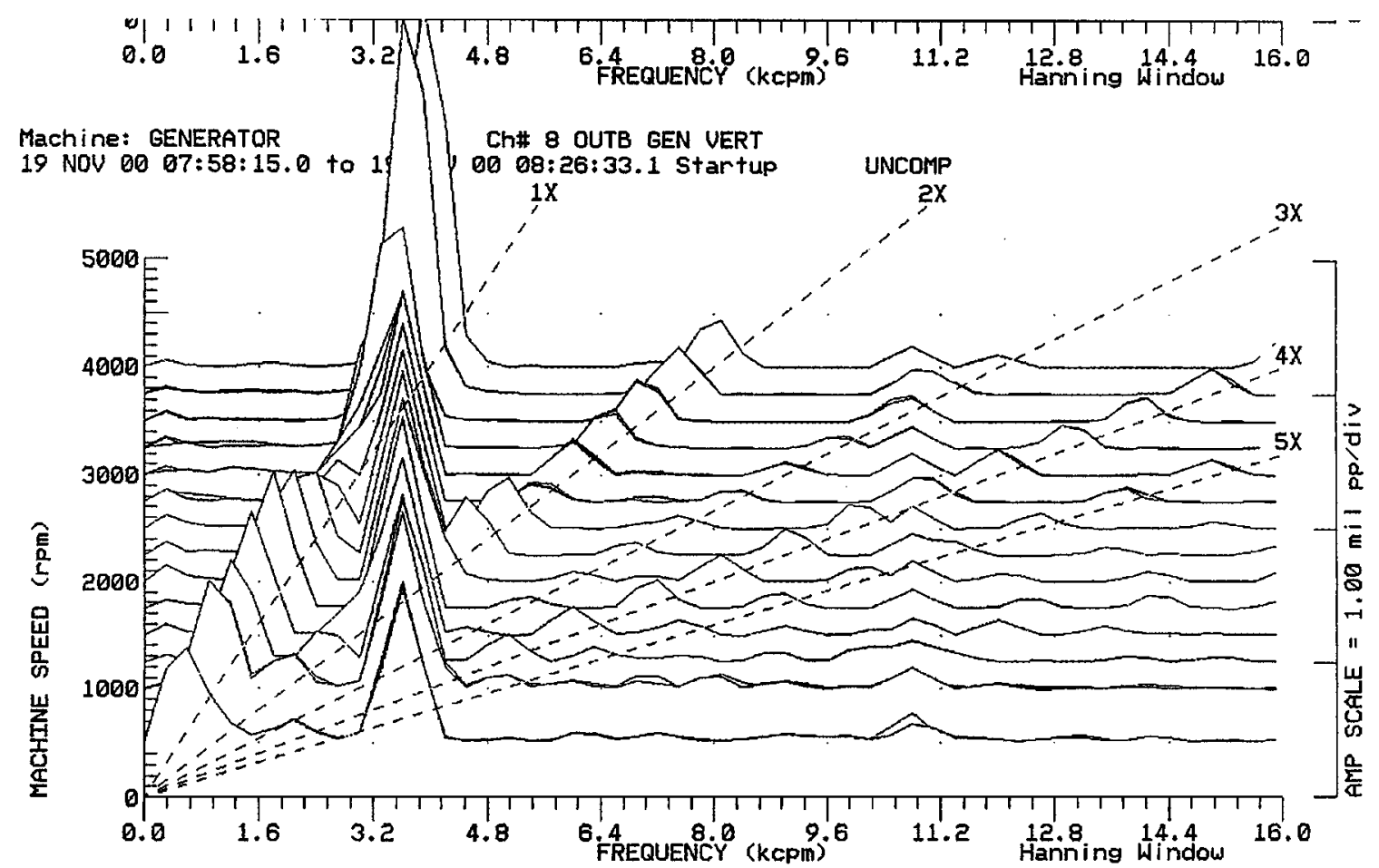

FIGURE 31

Generator spectrum, outboard vertical. 


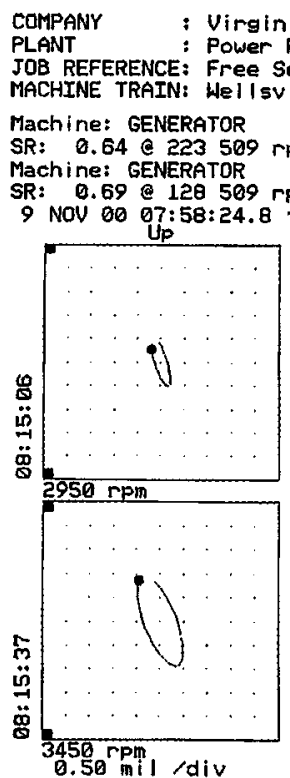

3450 rpmil /div ia Tech

Pivice

Ch\# 7 OUTB GEN HORZ

Ch\# 8 OUTB GEN VERT

19 NOV OD U8:27:09.5 Startup UP UP Filtered Comp

PLOT No.

135 deg.

45 deg.
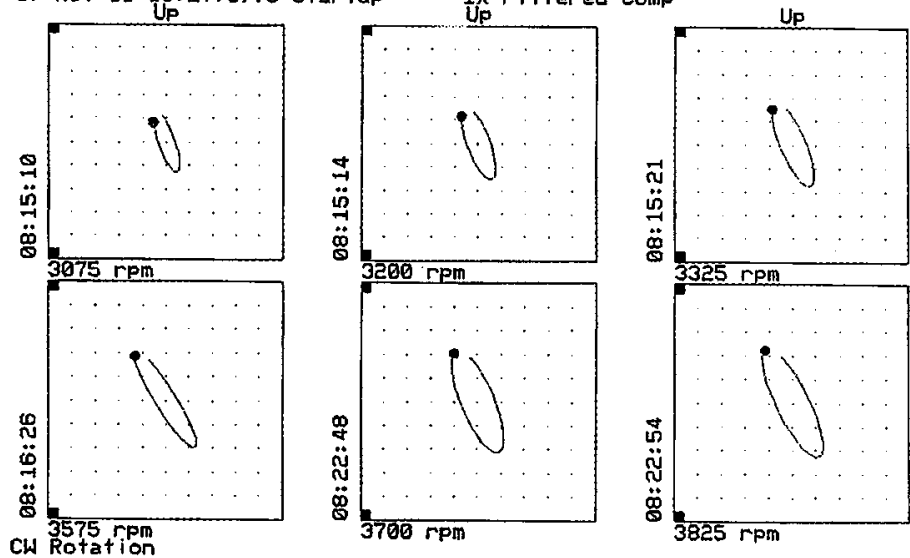

FIGURE 32

Generator orbit plots, outboard $1 \times$, compensated.
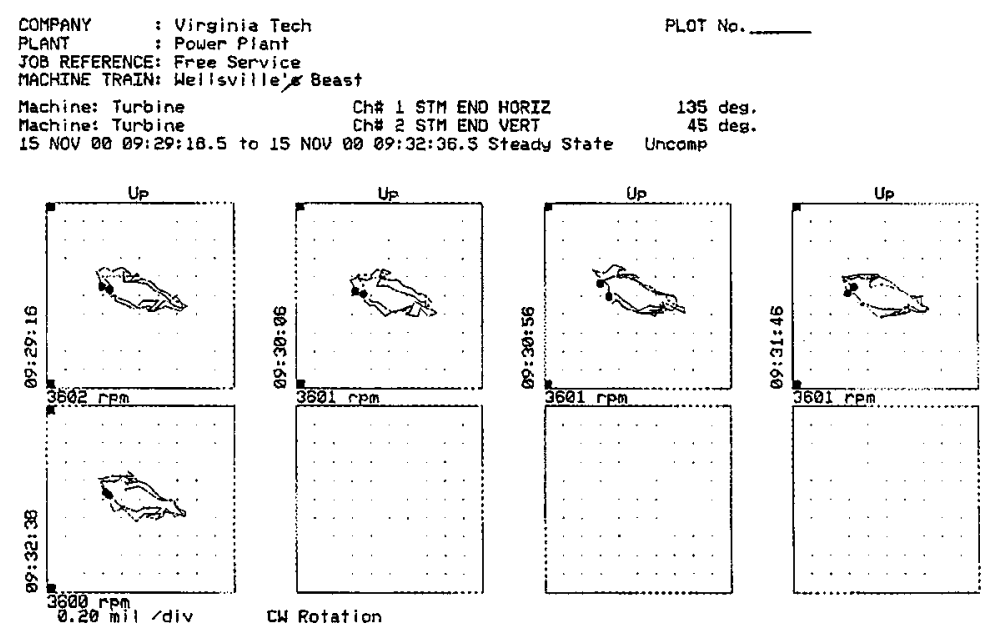

Machine: Turbine ChA 3 EXH END HORIZ

CW Rotation

15 Now eO 09:29: LE.5 to 15 NOW $0009: 32: 36.5$ steady state
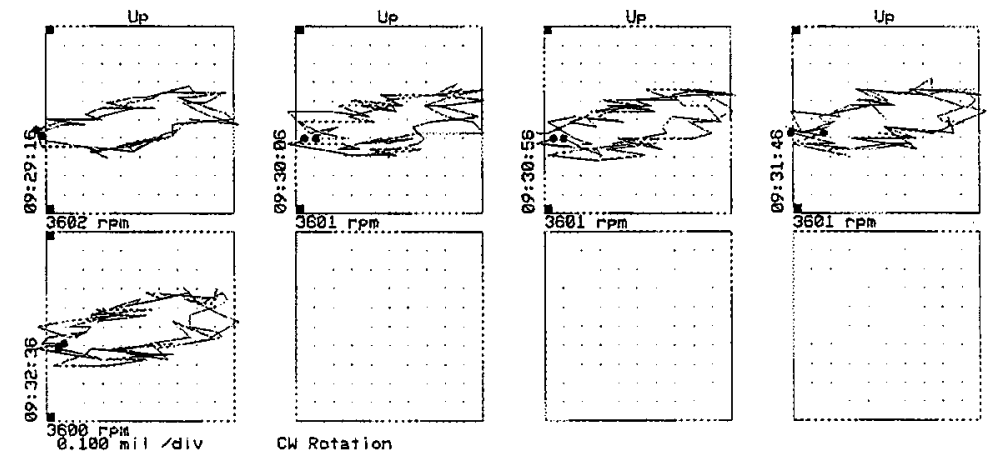

FIGURE 33

Turbine orbit plots, inboard and outboard, uncompensated. 

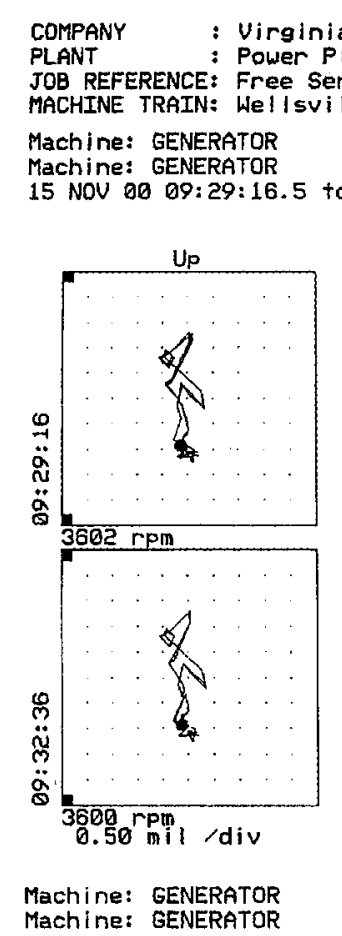
Machine: GENERATOR Ch\# 8 OUTB GEN VERT
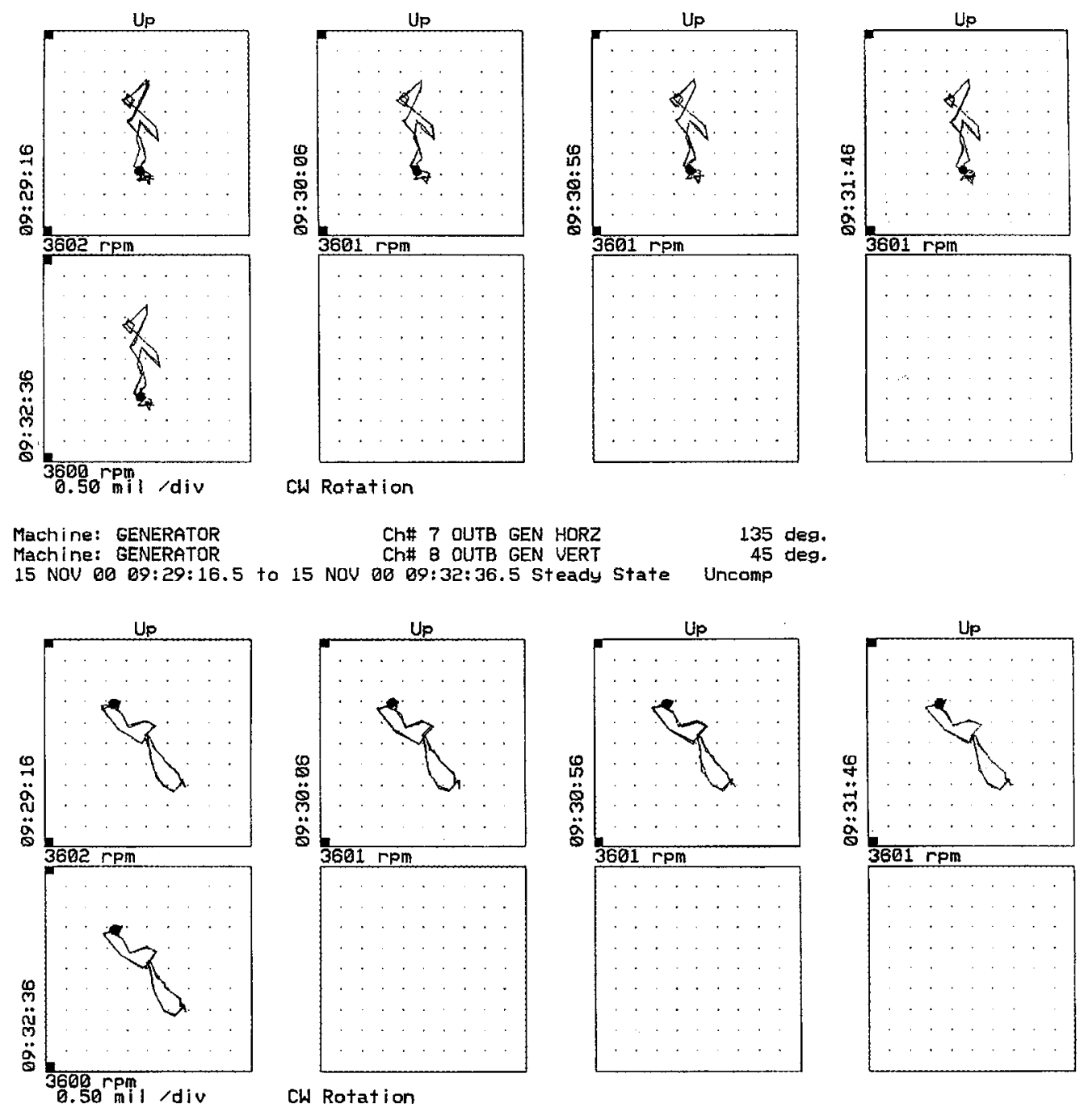

Ch\# 5 INB GEN HORZ $135 \mathrm{deg}$. Ch\# 6 INB GEN VERT

PLOT No. Uncomp deg. 
stated that the first real application was a success in that one of the knowledge-base files did indeed identify the source of the problem. Other problems were also identified, and they may continue to be a source of vibration. However, it is also true that the system identified potential problems that were proven not to exist. Obviously, the system would be a failure if only incorrect solutions were identified. This success of this first real application of the Virginia Tech's off-line expert system renews excitement about the potential power of such systems. It also points out the need for additional and continual update of the information in the current knowledge base.

The attempts to start a databank in the early 1980s were total failures due to lack of willingness or failure to grant permission for release of data by large companies. A request for data and machine-specific information resulted in only two replies and they both contained incomplete information. More recently, discussions were in progress to establish an Internet site to collect information and solve problems. The projected cost was substantial, so the progress was zero. Others have built expert system engines with no knowledge base. Little further progress has been reported.

The simple rules and conditions need to be improved to better determine specific problems and then build more complex rules and machine-specific rules. The task seems simple.

\section{REFERENCES}

Corsberg, D. 1987. Alarm filtering: practical control room upgrade using expert system concepts. Intech 34:39-43.

Durkin, J. 1996. Expert Systems: A view of the field. IEEE Expert Intelligent Systems and their Applications 11:56-63.

Hoglund, J. R. 1989. An expert system for off-line analysis of rotating equipment. (MS thesis, Virginia Polytechnic Institute and State University) Blacksburg, VA: Virginia Polytechnic Institute and State University.

Kirk, R. G., and Simpson, M. 1985. Full-Load testing of an 18,000 H.P. gas turbine driven centrifugal compressor for offshore platform service. Symposium on Stability, NASA CP-2409.
Kirk, R. G. 1986, November. Oil seal dynamics: considerations for analysis of centrifugal compressors, Proceedings of the 15th Turbomachinery Symposium. College Station, TX: Texas A\&M University.

Kirk, R. G. 2002, August. Condition monitoring and diagnosis of rotating machin. International Symposium on Machine Condition Monitoring and Diagnosis, Paper Presented at Annual Meeting of the Japan Society of Mechanical Engineers. Meijo University, Nagoya, Japan.

Kirk, R. G., Hoglund, J., and Keesee, J. 1989a. Application of artificial intelligence for rapid evaluation of turbomachinery dynamic response and stability. 149-154. Proceedings of the JSME International Symposium on Advanced Computers for Dynamics and Design. Tsuchiura, Japan:

Kirk, R. G., Hoglund, J., and Mondy, R. E. 1989c, September. Development of a PC-based off-line expert system for evaluation of turbomachinery response, 439-444. Proceedings of the 1st International Machinery Monitoring and Diagnostic Conference. Las Vegas, NV: Union College.

Kirk, R. G., Pawtowski, E. C., and Typrin, M. 1991, April. Intelligent filter for a PC-based expert system, 109-116. Proceedings of 45th Meeting of the Mechanical Failures Prevention Group. Annapolis, MD: .

Liddle, _., Lan, _., Reilly, .., and Steven, _. 1993. Diagnosing vibration problems with an expert system. Mechanical Engineering 54-55.

Pawtowski, E. C. 1996. Development of the Second-Generation IMTS (Intelligent Monitoring and Trending System) and WOT (Wizard of Tech) Expert System for Rotating Machinery. (MS thesis, Virginia Polytechnic Institute and State University).

Typrin, M., Pawtowski, E. C., and Kirk, R. G. 1992, April. A PC-based expert system for rotating machinery,-. International Conference on Rotordynamics. Venice, Italy : .

Typrin, M. 1992, February. IMTS (Intelligent Monitoring and Trending System): A PC-based Monitoring System for Rotating Machinery. (MS thesis, Virginia Polytechnic Institute and State University).

Vale, Z. A., and Mows, A. M. 1993, August. An expert system with temporal reasoning for alarm processing in power system control centers, 1307-1313. IEEE Transactions on Power Systems 8:3. 

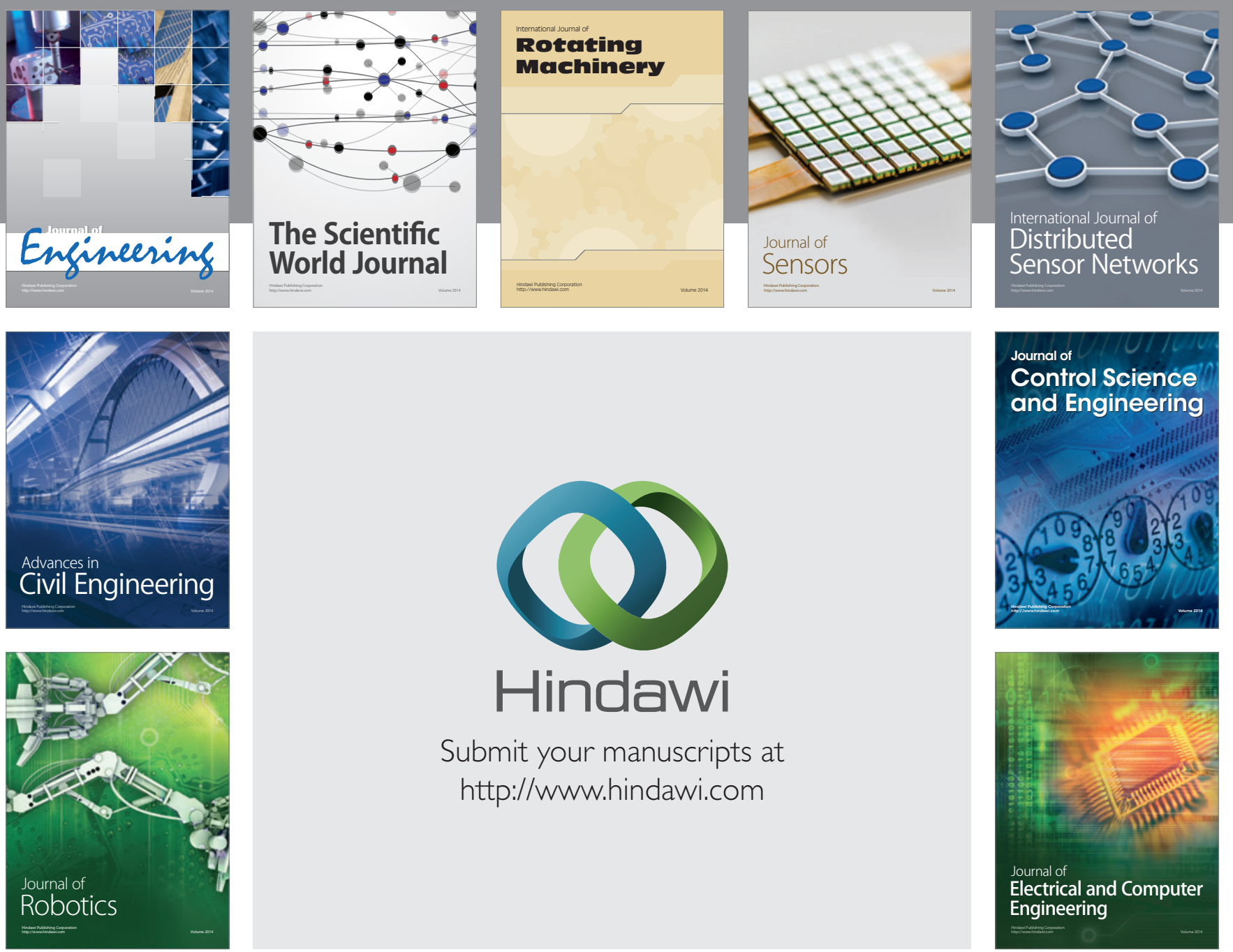

Submit your manuscripts at

http://www.hindawi.com
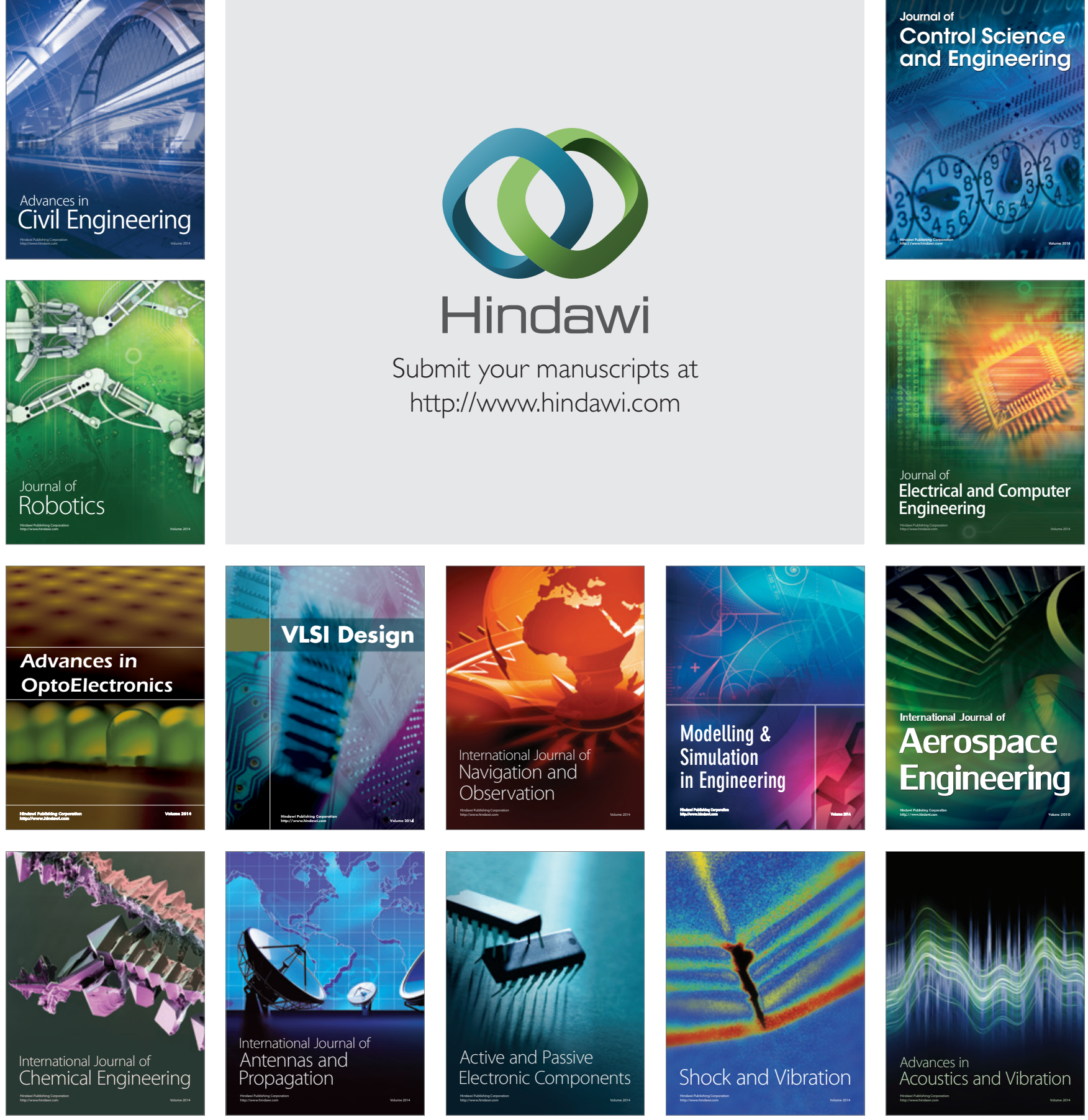